\title{
Psychometric properties of the Dutch Five Facet Mindfulness Questionnaire (FFMQ) in patients with fibromyalgia
}

\author{
Martine M. Veehof • Peter M. ten Klooster • Erik Taal • \\ Gerben J. Westerhof • Ernst T. Bohlmeijer
}

Received: 21 September 2010 /Revised: 17 December 2010 / Accepted: 10 January 2011 /Published online: 25 February 2011

(C) The Author(s) 2011. This article is published with open access at Springerlink.com

\begin{abstract}
Mindfulness-based interventions are increasingly being used in clinical populations to reduce psychological distress and improve functioning. The Five Facet Mindfulness Questionnaire (FFMQ) is a questionnaire that measures five facets of mindfulness: observe, describe, actaware, nonjudge and nonreact. The goal of this study was to examine the psychometric properties of the FFMQ in a clinical population of fibromyalgia patients. A total of 141 patients completed an online questionnaire on mindfulness (FFMQ) and theoretically related (e.g. acceptance, openness, alexithymia) and unrelated (physical health) constructs. Thirty-eight patients filled in the FFMQ twice. A confirmatory factor analysis (CFA) was conducted to test the five-factor structure of the FFMQ. Internal consistency and test-retest reliability were respectively assessed with Cronbach's $\alpha$ and intraclass correlation coefficients. Construct validity was examined by correlating FFMQ facets with theoretically related and unrelated constructs. Incremental validity in predicting mental health and psychological symptoms was examined with regression analyses. CFA confirmed the correlated five-factor structure of the FFMQ. Internal consistency of the five facets was satisfactory and test-retest reliability was good to excellent. Construct validity was excellent, as shown by the moderate to large correlations with related constructs (except observe facet) and weak correlation with a theoretically unrelated construct. Two of the five facets (actaware and nonjudge) had incremental validity over the others in predicting mental health and psychological symptoms. After controlling for related constructs, the actaware facet remained a significant
\end{abstract}

M. M. Veehof $(\bowtie) \cdot$ P. M. ten Klooster • E. Taal

G. J. Westerhof $\cdot$ E. T. Bohlmeijer

Department of Psychology, Health and Technology,

University of Twente,

P.O. Box 217, 7500 AE Enschede, The Netherlands

e-mail:m.m.veehof@utwente.nl predictor. This study showed satisfactory psychometric properties of the Dutch FFMQ in fibromyalgia patients. The observe facet, however, should be used with caution given its deviant relationship with theoretically related constructs.

Keywords Fibromyalgia $\cdot$ Five Facet Mindfulness Questionnaire - Mindfulness · Psychometric characteristics · Self-report assessment

\section{Introduction}

In recent years, there has been growing interest for mindfulness in the treatment of chronic pain and psychological distress. Mindfulness refers to a state of being attentive to and aware of experiences (including physical sensations, emotions, thoughts, imagery) occurring in the present moment in a nonjudgmental or accepting way $[1,2]$. It can be contrasted with a state of being caught up by memories, plans, fantasies or worries, and behaving on 'automatic pilot', without paying attention to one's actions [1]. Mindfulness, which is usually taught by a variety of meditation and/or attention exercises and psychoeducation, has been incorporated into several treatment programs. Well-known programs include mindfulness-based stress reduction [3], mindfulness-based cognitive therapy [4], dialectical behaviour therapy $[5,6]$, acceptance and commitment therapy [7] and acceptance-based behavioural therapy [8]. The effectiveness of these programs in reducing medical and psychological symptoms and improving quality of life has been shown in a wide range of clinical and nonclinical populations including patients with rheumatic conditions [8-14].

To get insight into the mechanisms that produce these beneficial effects, for example the mediating effects of 
different mindfulness facets, proper assessment of mindfulness is necessary. Several self-report measures of mindfulness have been developed, including the Freiburg Mindfulness Inventory [15], the Kentucky Inventory of Mindfulness Skills [16], the Mindful Attention Awareness Scale [1], the Cognitive and Affective Mindfulness Scale [17] and the Southampton Mindfulness Questionnaire [18]. Although these measures have shown satisfactory psychometric qualities [2], they differ in generalizability, content and structure, suggesting the lack of consensus among researchers about the operationalization of the construct mindfulness. Recently, Baer et al. have performed a study on the facet structure of mindfulness [2]. They combined the items of existing mindfulness questionnaires and conducted an exploratory factor analysis on all items. This analysis revealed five facets of mindfulness: (1) observing, defined in terms of noticing or attending to internal and external experiences; (2) describing, defined in terms of labelling internal experiences with words; (3) acting with awareness, defined in terms of attending to one's activities of the moment (opposite of acting on automatic pilot); (4) nonjudging of inner experience, defined in terms of taking a nonevaluative stance toward thoughts and feelings; and (5) nonreactivity to inner experience, defined in terms of allowing thoughts and feelings to come and go, without getting caught up in or carried away by them. The items with the highest loadings on these facets were selected and combined, resulting in a new mindfulness questionnaire: the Five Facet Mindfulness Questionnaire (FFMQ). The FFMQ has been tested in meditating and in student samples and has shown good psychometric properties [2, 19]. The FFMQ, however, has never been validated in a clinical sample, which is important given the wide implementation of mindfulness interventions in clinical populations. Moreover, test-retest reliability has never been investigated.

This study focused on the psychometric properties of the FFMQ in a clinical population of fibromyalgia patients. This is an important population in which mindfulness programs have been evaluated. Fibromyalgia is a common chronic pain syndrome of unknown aetiology. Given the psychological distress, which is often associated with this disease, mindfulness interventions seem especially appropriate for fibromyalgia. The beneficial effects of mindfulness interventions in fibromyalgia patients have been shown in several studies [20-23]. The aim of this study was to investigate the factor structure, reliability (internal consistency and test-retest reliability) and validity (construct validity and incremental validity) of the Dutch version of the FFMQ in patients with fibromyalgia. With regard to construct validity, both constructs incorporating elements of mindfulness (e.g. openness to experiences, acceptance) and constructs reflecting the absence of mindfulness (e.g. alexithymia, neuroticism) were included to assess convergent validity. Furthermore, constructs which were predicted to be weakly or not related to mindfulness (e.g. physical health) were used to assess discriminant validity. To determine the incremental validity, the relation of the different mindfulness facets with mental health and psychological symptoms (depression and anxiety) was assessed.

\section{Method}

Patients and procedure

From June 2009 till March 2010, participants were recruited via the website of the Dutch Fibromyalgia Patient Association. Inclusion criterion was a self-reported diagnosis of fibromyalgia, confirmed by a doctor. If participants met this criterion, they were asked to complete an online questionnaire on mindfulness and theoretically related and unrelated constructs. Till August 2009, patients were asked if they were willing to complete the FFMQ for a second time. Patients who agreed received an email with a hyperlink to this second questionnaire, 2 weeks after completion of the first questionnaire.

\section{Measures}

\section{Patient characteristics}

Questions were included regarding age, gender, education (low: primary school, lower vocational education; intermediate: secondary school, intermediate vocational education; high: higher vocational training, university), disease duration and average pain intensity. The latter was measured on an 11-point numerical rating scale (NRS) ranging from 'no pain' (0) to 'unbearable pain' (10).

\section{$F F M Q$}

The FFMQ is a 39-item questionnaire that measures five facets of mindfulness: observe (8 items), describe ( 8 items), actaware ( 8 items), nonjudge ( 8 items) and nonreact (7 items) [2]. Participants were asked to rate the degree to which several statements were true for them. Items were scored on a five-point Likert scale ranging from 1 (never or very rarely true) to 5 (very often or always true). Facet scores were computed by summing the scores on the individual items. Facet scores range from 8 to 40 (except for the nonreact facet which ranges from 7 to 35), with higher scores indicating more mindfulness. The Dutch FFMQ has shown adequate psychometric properties in meditating and psychology student samples (unpublished data).

Since the Dutch FFMQ has only been evaluated in highly educated people and in people with meditation experience, the questionnaire was first cognitively pretested 
in a clinical sample of 11 rheumatic patients to investigate the clarity of the wording of the items. In this pretest, patients filled in the FFMQ while they were thinking aloud. Afterwards, they were interviewed, using the three-step test-interview method [24], on the problems they perceived. Fourteen items (see Table 3) appeared problematic and each facet contained at least one problematic item. These items were partially rephrased by an experienced mindfulness psychologist without changing the content or meaning of the items. For example, 'I criticize myself for having irrational or inappropriate emotions' was changed to 'I criticize myself for having illogical or inappropriate emotions'. And 'I perceive my feelings and emotions without having to react to them' was changed to 'I perceive my feelings and emotions without having to do something with them'.

\section{Acceptance and Action Questionnaire-II (AAQ-II)}

The 10-item AAQ-II was used to assess the ability to accept aversive internal experiences, like negative emotions, thoughts and memories and to pursue goals in the presence of these experiences [25]. Participants were asked to rate on a seven-point Likert scale the degree to which several statements were true for them. A total score, ranging from 10 to 70 , was computed by summing the scores on the individual items. Higher scores indicate higher levels of general acceptance and less experiential avoidance. The Dutch AAQ-II has shown adequate psychometric properties [25]. Internal consistency, expressed with Cronbach's $\alpha$ coefficient, in our study was 0.88 .

\section{NEO Five-Factor Inventory (NEO-FFI)}

Neuroticism (tendency to experience negative emotions) and openness to new experiences (proactive seeking and appreciation of new experiences) were measured using two scales of the NEO-FFI, a questionnaire addressing five core personality traits: neuroticism, extraversion, openness to experiences, conscientiousness and agreeableness [26-28]. Both the neuroticism and the openness to experiences scale consist of 12 statements. Participants were asked to rate the degree to which they agree with these statements. Each statement was rated on a five-point scale, yielding a scale score ranging from 12 to 60 . Higher scores reflect higher levels of neuroticism or openness to experiences. The Dutch NEO-FFI has shown good psychometric properties [27]. Cronbach's $\alpha$ coefficients for neuroticism and openness to experiences in this study were respectively 0.91 and 0.77 .

\section{Toronto Alexithymia Scale (TAS-20)}

The TAS-20 was used to assess alexithymia $[29,30]$. The TAS-20 is a 20 -item questionnaire containing three factors reflecting distinct facets of alexithymia: (1) difficulty in identifying feelings (7 items), (2) difficulty in describing feelings (5 items) and (3) externally oriented thinking (8 items). Items are scored on five-point scale, ranging from strongly disagree (1) to strongly agree (5). A total score was computed by summing the scores on all items. Total score ranges from 20 to 100 with higher scores indicating more alexithymia. The Dutch TAS-20 has shown good psychometric properties [31]. Internal consistency of the TAS-20 in this study was satisfactory (Cronbach's $\alpha=0.83$ ).

\section{Hospital Anxiety and Depression Scale (HADS)}

The HADS was used to measure the presence and severity of anxiety and depression symptoms [32]. The HADS is a 14-item questionnaire comprising two subscales: the HADS-A (7 items) measuring anxiety and the HADS-D (7 items) measuring depression. Participants were asked to rate the degree to which they experienced several emotions in the past week. All items were rated on a four-point scale $(0-3)$. Scale scores were computed by summing the scores on the individual items of a subscale. Scale scores range from 0 to 21 with higher scores indicating more symptomatology. The Dutch HADS has shown good psychometric properties [33]. Cronbach's $\alpha$ coefficients of the HADS-A and HADS-D in this study were respectively 0.88 and 0.86 .

\section{Short-Form 12-Item Health Survey (SF-12)}

The SF-12, version 2, was used to assess mental and physical health [34]. The SF-12 is a shortened form of the SF-36 and contains items on physical functioning (2 items), role limitations because of physical health problems ( 2 items), bodily pain $(1$ item), general health perceptions (1 item), vitality (1 item), social functioning (1 item), role limitations because of emotional problems ( 2 items) and general mental health (2 items). Norm-based physical component summary (PCS) and mental component summary (MCS) scores were calculated, ranging from 0 (poor well-being) to 100 (excellent well-being), with a mean of 50 and a standard deviation of 10 in the general population of the USA [35]. The SF-12 has shown good psychometric properties [34, 36]. Internal consistency of the SF-12 in this study was satisfactory with a Cronbach's $\alpha$ coefficient of 0.79 for both components.

\section{Data analysis}

Descriptive and standard psychometric analyses were performed using the statistical packages SPSS16. Missing values were imputed using the median value of an item (if no more than $10 \%$ of the items of a (sub)scale were missing). Given the normal distribution of the data, tested with Kolmogorov-Smirnov tests, parametric tests were 
used. Confirmatory factor analysis (CFA) was conducted with LISREL 8.70 (Scientific Software International, Lincolnwood, IL, USA). Missing values were imputed using the expectation-maximization algorithm procedure.

\section{Factor structure}

A CFA using the maximum likelihood estimation procedure was conducted to test the five-factor structure of the FFMQ. We first tested a correlated five-factor model. This model provided the best fit in the original validations of the FFMQ and assumes that the scale measures five distinct, but related, facets of mindfulness. The items were constrained to load on one factor only, error terms were not allowed to correlate and the variance of the factors was fixed to 1 . Second, we tested a hierarchical five-factor model, in which the five factors were indicators of an overall mindfulness factor.

Besides the overall model chi-square statistic, where smaller values indicate better fit, multiple indices were used to examine the fit of the models. As suggested by $\mathrm{Hu}$ and Bentler [37], the non-normed fit index (NNFI), the comparative fit index (CFI), the standardized root mean square residual (SRMR), and the root mean square error of approximation (RMSEA) were used. NNFI and CFI values $\geq 0.90$ and 0.95 were considered indicative of acceptable and good model fit, respectively. For the SRMR and RMSEA, values $\leq 0.10$ and 0.08 and $\leq 0.08$ and 0.06 , respectively, were considered to reflect acceptable and good fit $[38,39]$.

Additionally, Pearson intercorrelations were computed between the five facets. Because the facets are assumed to measure related but distinct constructs, it was hypothesized that the facets should be significantly, but only moderately, correlated $(r=0.30-0.50)$.

\section{Internal consistency and test-retest reliability}

Internal consistency of the FFMQ facets was assessed with Cronbach's $\alpha$ coefficients. Values above 0.70 were considered acceptable for research purposes [40]. Test-retest reliability was assessed with intraclass correlation coefficients (ICCs) using a two-way random effects model and an absolute agreement definition. Single measures ICCs are reported. Values $<0.40$ were interpreted as poor, values between 0.40 and 0.59 as fair, values between 0.60 and 0.74 as good and values $>0.75$ as excellent [40].

\section{Construct validity}

Pearson correlation coefficients were calculated between FFMQ facets and other constructs, which were predicted to be moderately or strongly related (convergent validity) or weakly or not related (discriminant validity) to mindfulness.
Correlations between 0.50 and 1.00 were interpreted as strong, correlations between 0.30 and 0.50 as moderate, correlations between 0.10 and 0.30 as small and correlations $<0.10$ as weak [41]. Based on the content of the mindfulness facets and the other constructs, as well as the results of Baer et al. [2], predictions were made about the direction and strength of the correlations. Furthermore, predictions were made about which mindfulness facet(s) should most strongly correlate with each construct.

At least moderate and positive correlations were predicted between mindfulness facets (except observe facet) and acceptance (AAQ-II). Because acceptance includes the nonjudgmental acceptance of inner experiences, the strongest correlation was expected with the nonjudge facet. At least a moderate and positive correlation was also expected between the observe facet and openness to experiences (NEO-FFI), which includes the ability to be attentive to experiences. Similar to the results of Baer et al. [2], weak to small correlations were expected between openness to experiences and the other mindfulness facets (describe, actaware, nonjudge, nonreact). At least moderate but negative correlations were expected between mindfulness facets (except observe facet) and alexithymia (TAS-20). Alexithymia includes the difficulty to identify and describe feelings. Therefore, the strongest correlation was expected with the describe facet. At least moderate and negative correlations were also predicted between mindfulness facets (except observe facet) and neuroticism (NEO-FFI), anxiety (HADS-A) and depression (HADS-D). Previous studies $[2,16,19]$ have shown that these psychological constructs/ symptoms were most strongly correlated with the actaware and nonjudge facets. Therefore, the strongest correlations were expected with these facets. With regard to physical health (SF-12 PCS), weak correlations were expected with mindfulness facets. This construct does not seem to incorporate elements of mindfulness, neither reflects the absence of mindfulness.

Recently, Baer et al. have shown that the observe facet is sensitive to changes with meditation experience that alters its relationship with other variables [2, 19]. Only when internal and external experiences are observed mindfully, which is a skill that can be developed with practice, the observe facet acts like the other facets and is positively related with positive psychological functioning. Participants of this study were recruited via the website of the Dutch Fibromyalgia Patient Association. Generally, we do not expect a considerable amount of mindfulness experience in this study sample. Therefore, nonsignificant and weak correlations were expected between the observe facet and acceptance (AAQ-II), alexithymia (TAS-20), neuroticsm (NEO-FFI), anxiety (HADS-A) and depression (HADS-D). 


\section{Incremental validity}

Regression analyses (method forced entry) were conducted to examine whether the FFMQ facets contribute independently to the prediction of mental health (SF-12 MCS), depression (HADS-D) and anxiety (HADS-A), after controlling for differences in acceptance, alexithymia, neuroticism and openness to new experiences. In the first step, the five mindfulness facets were entered. In the second step, acceptance, alexithymia, neuroticism and openness to new experiences were entered.

\section{Results}

A total of 192 patients responded and agreed to participate. Fifty-one (27\%) of them did not complete at least the FFMQ or had too much missing values. A total of 141 patients were included for analysis. These patients did not significantly differ from the patients that were excluded with regard to patient characteristics. Mean age of the patients was 43.1 (SD 10.9) years and most of them (93\%) were female. Patient characteristics as well as scores on FFMQ and other questionnaires are summarized in Table 1.

\section{Factor structure}

Results of the CFA are shown in Tables 2 and 3. All fit indices, except the SRMR in the hierarchical model, met the recommended criteria of acceptable model fit (Table 2). As expected, the correlated five-factor structure provided the best model fit. In this model, high factor loadings (all between 0.54 and 0.84 ) were found for the facets describe, actaware and nonjudge (Table 3). The observe (items 1 and 11) and nonreact (items 4 and 21) facets contained some items with factor loadings below the permissible minimum of 0.40 advocated in factor analysis [42]. In the hierarchical five-factor model, we found comparable factor loadings (data not shown). In this model, the observe facet (0.35) loaded lower on an overall mindfulness construct than the describe $(0.68)$, actaware (0.70), nonjudge (0.57) and nonreact $(0.70)$ facets.

Correlations (two-tailed) between the mindfulness facets (except the observe facet) were significant $(p<0.01)$ and ranged from 0.29 to 0.52 , suggesting that they represent related but distinct constructs. The observe facet correlated nonsignificantly $(p>0.05)$ with the actaware $(r=0.05)$ facet and significantly $(p<0.01)$ with the describe $(r=0.29)$ and nonreact $(r=0.30)$ facets. A small but significant $(p<0.05)$ negative correlation was found with the nonjudge facet $(r=-0.20)$.
Table 1 Patient characteristics and scores on outcome measures

\begin{tabular}{ll}
\hline Patient characteristics & Values $^{\mathrm{a}}$ \\
\hline Age, years $(n=140)$ & $43.1(10.9)$ \\
Gender (\% female) $(n=140)$ & 93 \\
Educational level $(n=139)$ & 18 \\
Low (\%) & 57 \\
Intermediate (\%) & 25 \\
High (\%) & $9.4(8.7)$ \\
Disease duration, years $(n=141)$ & $6.7(1.6)$ \\
Pain (NRS; 0-10) $(n=137)$ & \\
Mindfulness (FFMQ; $n=141)$ & $27.7(4.6)$ \\
Observe (0-40) & $27.3(6.5)$ \\
Describe (0-40) & $25.1(6.3)$ \\
Actaware (0-40) & $26.4(6.8)$ \\
Nonjudge (0-40) & $21.7(4.2)$ \\
Nonreact (0-35) & $46.3(10.4)$ \\
Acceptance (AAQ-II; $10-70 ; n=132)$ & $50.6(10.7)$ \\
Alexithymia (TAS-20; 20-100; $n=131)$ & $35.8(10.7)$ \\
Neuroticism (NEO-FFI; $12-60 ; n=131)$ & $40.6(6.7)$ \\
Openness to experiences $(\mathrm{NEO-FFI;} 12-60 ; n=131)$ & $6.5(4.3)$ \\
Depression (HADS-D; $0-21 ; n=132)$ & $8.4(4.7)$ \\
Anxiety (HADS-A; $0-21 ; n=132)$ & $40.4(9.9)$ \\
Mental health (SF-12 MCS; $0-100 ; n=139)$ & $31.3(7.8)$ \\
Physical health (SF-12 PCS; $0-100 ; n=139)$ & \\
\hline
\end{tabular}

$N R S$ numerical rating scale, $F F M Q$ Five Facet Mindfulness Questionnaire, AAQ-II Acceptance and Action Questionnaire-II, TAS-20 Toronto Alexithymia Scale, NEO-FFI NEO Five-Factor Inventory, $H A D S-D$ Hospital Anxiety and Depression Scale-Depression, $H A D S-A$ Hospital Anxiety and Depression Scale-Anxiety, $S F-12$ $M C S$ Short-Form 12-Item Health Survey mental component summary, SF-12 PCS Short-Form 12-Item Health Survey physical component summary

${ }^{a}$ Values are means (SD) unless otherwise indicated

\section{Reliability}

Results of the reliability analyses are shown in Table 4 . Internal consistency of the FFMQ facets, measured with Cronbach's $\alpha$ coefficient, was sufficient, ranging from 0.69 (observe) to 0.90 (describe). Deletion of the items with low item-total correlations did not change the coefficients substantially.

Of 96 patients (included till August 2009), 55 were willing to fill in the FFMQ for a second time. Eventually, 38 patients really completed the FFMQ twice. These 38 patients did not significantly differ from the patients who did not fill in the FFMQ for a second time with regard to patient characteristics. On the actaware facet, however, these patients scored significantly lower (22.9 versus 27.6 ), indicating that they were less mindful on this facet. Testretest reliability of the FFMQ facets was good to excellent with single measure ICCs ranging from 0.61 (actaware) to 
Table 2 Goodness of fit of the FFMQ in patients with fibromyalgia $(n=146)$

\begin{tabular}{lllllll}
\hline & $\chi^{2}$ & $d f$ & NNFI & CFI & SRMR & RMSEA (90\% CI) \\
\hline Correlated five-factor model & $1,339.338$ & 692 & 0.901 & 0.908 & 0.098 & $0.080(0.074-0.087)$ \\
Hierarchical five-factor model & $1,395.213$ & 697 & 0.898 & 0.904 & 0.111 & $0.083(0.077-0.089)$
\end{tabular}

$\chi^{2}$ normal theory weighted least squares chi-square, $d f$ degrees of freedom, NNFI non-normed fit index, $C F I$ comparative fit index, SRMR standardized root mean square residual, RMSEA root mean square error of approximation ( $90 \%$ confidence interval)

0.84 (nonjudge). Paired samples $t$ tests showed no significant differences between the scores at both assessment times.

\section{Construct validity}

In Table 5, correlations between mindfulness facets and theoretically related or unrelated constructs are shown. As expected, moderate to large correlations were found between mindfulness facets (except observe facet) and variables that were predicted to be related to mindfulness (acceptance, alexithymia, neuroticism, depression, anxiety). Nonsignificant and generally weak correlations were found between these variables and the observe facet, except for alexithymia which showed a small but significant correlation with the observe facet. A large correlation was found between the observe facet and openness to experiences. The other FFMQ facets showed weak to small correlations with this construct, except for the describe facet which was moderately correlated. Conforming to our predictions, generally weak and nonsignificant correlations were found between mindfulness facets and physical health. All correlations were in the expected direction, and hypotheses about which facets should most strongly correlate with each construct were confirmed.

\section{Incremental validity}

Results of the regression analyses (see Table 6) showed that two (actaware and nonjudge) of the five FFMQ facets were significant predictors and had incremental validity over the others in the prediction of mental health, depression and anxiety. Furthermore, the nonreact facet appeared a significant predictor of anxiety. All mindfulness facets together predicted respectively $44 \%, 38 \%$ and $57 \%$ of the total variation in mental health, depression and anxiety. After controlling for differences in alexithymia, acceptance, neuroticism and openness to new experiences, the actaware facet remained significantly associated with mental health, depression and anxiety.

\section{Discussion}

The aim of this study was to investigate the psychometric properties of the Dutch FFMQ in patients with fibromyalgia.
Factor structure, internal consistency, test-retest reliability, construct validity and incremental validity in predicting mental health, depression and anxiety were examined. In general, the results of our study were promising and satisfactory.

CFA showed acceptable model fit for a correlated fivefactor structure of the FFMQ. This result is in accordance with the results of the original validations of the FFMQ $[2,19]$ and assumes that the FFMQ measures five distinct, but related facets of mindfulness. Model fit was less when a hierarchical five-factor structure was used. This is not surprising given the poor loading of the observe facet on the overall mindfulness construct. The latter might be explained by the lack of meditation experience in our study sample $[2,19]$.

Although the model fit for a correlated five-factor structure was acceptable, the observe (items 1 and 11) and nonreact (items 4 and 21) facets contained some items with low factor loadings $(<0.40)$, meaning that these items contributed minimally to their underlying facet. Nevertheless, internal consistency of these facets, expressed with Cronbach's $\alpha$ coefficient, is considered sufficient for research purposes. These low factor loadings, compared with the findings of Baer et al. [2], might be explained by cultural differences or differences between the study samples (psychology students and experienced meditators versus fibromyalgia patients). Future research should address whether these items will also be problematic in other clinical and nonclinical populations and if adaptation of the FFMQ is necessary.

This is the first study that examined the test-retest reliability of the FFMQ. Our analyses showed good to excellent results. Findings are in accordance with the results reported on the test-retest reliability of other mindfulness questionnaires, like the KIMS [16] and the MAAS [1].

Construct validity was excellent, as shown by the correlations between mindfulness facets and theoretically related (e.g. openness to experiences, acceptance, alexithymia and neuroticism) and unrelated (physical health) constructs. All correlations were in the expected direction and strength, except for the correlation between the describe facet and openness to experiences, which was moderate $(r=0.35)$ instead of small $(r<0.30)$, and the correlation between the observe facet and alexithymia, which was small $(r=-0.24)$ instead of weak $(-0.10>r<0.10)$. Theoretically, these findings are not surprising. Compared to the findings of Baer et 
Table 3 Completely standardized factor loadings (LISREL) in the correlated five-factor model of the FFMQ $(n=146)$

\begin{tabular}{|c|c|c|c|c|c|}
\hline & Observe & Describe & Actaware & Nonjudge & Nonreac \\
\hline Item 1 & 0.12 & & & & \\
\hline Item 2 & & 0.80 & & & \\
\hline Item $3^{a}$ & & & & 0.62 & \\
\hline Item $4^{\mathrm{a}}$ & & & & & 0.17 \\
\hline Item $5^{\mathrm{a}}$ & & & 0.75 & & \\
\hline Item 6 & 0.41 & & & & \\
\hline Item 7 & & 0.70 & & & \\
\hline Item 8 & & & 0.67 & & \\
\hline Item $9^{a}$ & & & & & 0.56 \\
\hline Item 10 & & & & 0.66 & \\
\hline Item $11^{\mathrm{a}}$ & 0.23 & & & & \\
\hline Item 12 & & 0.81 & & & \\
\hline Item 13 & & & 0.81 & & \\
\hline Item $14^{\mathrm{a}}$ & & & & 0.54 & \\
\hline Item $15^{\mathrm{a}}$ & 0.66 & & & & \\
\hline Item 16 & & 0.74 & & & \\
\hline Item 17 & & & & 0.65 & \\
\hline Item $18^{\mathrm{a}}$ & & & 0.71 & & \\
\hline Item $19^{\mathrm{a}}$ & & & & & 0.56 \\
\hline Item $20^{\mathrm{a}}$ & 0.54 & & & & \\
\hline Item 21 & & & & & 0.37 \\
\hline Item $22^{\mathrm{a}}$ & & 0.69 & & & \\
\hline Item 23 & & & 0.70 & & \\
\hline Item 24 & & & & & 0.68 \\
\hline Item 25 & & & & 0.82 & \\
\hline Item 26 & 0.72 & & & & \\
\hline Item 27 & & 0.70 & & & \\
\hline Item 28 & & & 0.58 & & \\
\hline Item $29^{\mathrm{a}}$ & & & & & 0.44 \\
\hline Item 30 & & & & 0.83 & \\
\hline Item 31 & 0.78 & & & & \\
\hline Item 32 & & 0.61 & & & \\
\hline Item 33 & & & & & 0.86 \\
\hline Item 34 & & & 0.74 & & \\
\hline Item $35^{\mathrm{a}}$ & & & & 0.81 & \\
\hline Item 36 & 0.42 & & & & \\
\hline Item 37 & & 0.84 & & & \\
\hline Item 38 & & & 0.74 & & \\
\hline Item $39^{\mathrm{a}}$ & & & & 0.79 & \\
\hline
\end{tabular}

\footnotetext{
${ }^{a}$ Wording of item is changed based on the results of a cognitive pretest in a clinical population
}

al. [2], these correlations are remarkable however. With respect to these findings, we have to note that generally all correlations between mindfulness facets and theoretically related constructs in the study of Baer et al. [2] were lower than the correlations we found. Differences might be explained by cultural differences, differences in the study
Table 4 Results of the reliability analyses of the FFMQ

\begin{tabular}{lcc}
\hline FFMQ facets & $\begin{array}{l}\text { Internal consistency } \\
\text { Cronbach's } \alpha\end{array}$ & $\begin{array}{l}\text { Test-retest reliability } \\
\text { ICC }(95 \% \text { CI })\end{array}$ \\
\hline Observe & 0.69 & $0.73(0.53-0.85)$ \\
Describe & 0.90 & $0.84(0.70-0.92)$ \\
Actaware & 0.89 & $0.61(0.36-0.78)$ \\
Nonjudge & 0.89 & $0.84(0.70-0.91)$ \\
Nonreact & 0.74 & $0.71(0.50-0.84)$ \\
\hline ICC intraclass correlation coefficient $95 \% C I 95 \%$ confidence interval
\end{tabular}

sample (psychology students versus fibromyalgia patients) or differences in meditation experience. Hypotheses about which facets should most strongly correlate with each construct were confirmed and in accordance with the results of Baer et al. [2].

Regression analyses showed that the actaware and nonjudge facets had incremental validity over the others in the prediction of mental health, depression and anxiety. Furthermore, the nonjudge facet appeared a significant predictor of anxiety. These facets independently explained a proportion of the variance that was not explained by the others. This finding is in accordance with the results of Baer et al. [2]. The actaware facet seemed to be the most important facet in the prediction of mental health and psychological symptoms. This facet remained significantly related with mental health, depression and anxiety $(p<0.01)$ after controlling for acceptance, neuroticism, openness and alexithymia. The other facets lost their significance when these variables were added to the model. All facets together explained respectively $44 \%, 38 \%$ and $57 \%$ of the variance in mental health, depression and anxiety, indicating that mindfulness is highly related with mental health and psychological symptoms. The direction of this relationship, however, cannot be deduced from the results of our crosssectional study. Future research should address the causality of the relationship with mental health and psychological symptoms in a longitudinal study design.

This is the first study that validated the FFMQ in a clinical population. Facet scores were higher than the scores reported by Baer et al. [19] in a community sample. At present, norm scores for a Dutch community sample are not available yet. Therefore, it was not possible to make a statistical comparison between both samples. Future research should reveal if mindfulness scores are really higher in clinical sample compared to a community sample.

A limitation of this study is that we did not assess the amount of meditation experience of the participants. Baer et al. showed that the factor structure of the FFMQ and the relationship of the observe facet with theoretically related constructs change as a function of meditation experience $[2,19]$. Only in participants with meditation experience the 
Table 5 Correlations between mindfulness facets and other constructs $(n=131-139)$

NEO-FFI NEO Five-Factor Inventory, AAQ-II Acceptance and Action Questionnaire-II, TAS-20 Toronto Alexithymia Scale, HADS-D Hospital

Anxiety and Depression

Scale-Depression, HADS-A

Hospital Anxiety and

Depression Scale-Anxiety, SF-12 PCS Short-Form 12-Item

Health Survey physical

component summary

$* * p \leq 0.01$

\begin{tabular}{lccccc}
\hline \multirow{2}{*}{ Construct } & \multicolumn{2}{l}{ FFMQ facet } & & \\
\cline { 2 - 5 } & Observe & Describe & Actaware & Nonjudge & Nonreact \\
\hline Predicted positive correlations & & & & & \\
Openness to experiences (NEO-FFI) & $0.56^{* *}$ & $0.35^{* *}$ & 0.12 & -0.08 & $0.28^{* *}$ \\
Acceptance (AAQ-II) & -0.08 & $0.44^{* *}$ & $0.50^{* *}$ & $0.68^{* *}$ & $0.45^{* *}$ \\
Predicted negative correlations & & & & & \\
Alexithymia (TAS-20) & $-0.24^{* *}$ & $-0.73^{* *}$ & $-0.55^{* *}$ & $-0.51^{* *}$ & $-0.40^{* *}$ \\
Neuroticism (NEO-FFI) & 0.10 & $-0.41^{* *}$ & $-0.67^{* *}$ & $-0.67^{* *}$ & $-0.45^{* *}$ \\
Depression (HADS-D) & 0.01 & $-0.32^{* *}$ & $-0.54^{* *}$ & $-0.49^{* *}$ & $-0.37^{* *}$ \\
Anxiety (HADS-A) & 0.05 & $-0.38^{* *}$ & $-0.66^{* *}$ & $-0.60^{* *}$ & $-0.50^{* *}$ \\
Predicted no correlation & & & & & -0.01 \\
Physical health (SF-12 PCS) & -0.10 & 0.13 & -0.06 & -0.06 \\
\hline
\end{tabular}

observe facet becomes a clear facet of mindfulness and acts like the other facets in relation to other constructs. Our study sample was recruited by self-selection, which might have led to the selection of patients with interest for and/or experience with mindfulness meditation. Nevertheless, we do not expect that the participants had a significant amount of meditation experience, since our results were largely in concordance with the findings of Baer et al. in a predominantly non-meditating student sample [2]. In future research, the influence of meditation experience should be assessed. Another point concerns the generalizability of the results. Participants seemed representative for the average group of fibromyalgia patients regarding age and gender. Results, however, should be generalized with caution to other groups of clinical patients as well as experienced meditators. Finally, results were obtained with a modified version of the FFMQ. Although differences with the original version of the FFMQ were minimal and the meaning of the items was not changed, results should be generalized with caution to the original translation of the Dutch FFMQ.

All in all, this study shows that the FFMQ is a reliable and valid measure to assess mindfulness in patients with fibromyalgia. With the current study design, it was not possible to investigate sensitivity to change. Future studies should reveal if the FFMQ is also sensitive to detect changes over time. Finally, our results support the multifaceted structure of mindfulness, as earlier suggested [2],
Table 6 Results of regression analyses showing prediction of mental health (SF-12 MCS), depression (HADS-D) and anxiety (HADS-A) by mindfulness facets and other constructs

\begin{tabular}{|c|c|c|c|c|c|c|c|c|c|}
\hline \multirow[t]{2}{*}{ FFMQ facets } & \multicolumn{3}{|c|}{ Mental health } & \multicolumn{3}{|c|}{ Depression } & \multicolumn{3}{|c|}{ Anxiety } \\
\hline & Beta & $t$ & Sign & Beta & $t$ & sign & Beta & $t$ & Sign \\
\hline \multicolumn{10}{|l|}{ Step 1} \\
\hline Observe & -0.08 & -0.99 & 0.33 & 0.02 & 0.23 & 0.82 & 0.07 & 1.08 & 0.28 \\
\hline Describe & 0.08 & 1.00 & 0.32 & -0.08 & -0.94 & 0.35 & -0.09 & -1.31 & 0.19 \\
\hline Actaware & 0.42 & 5.10 & 0.00 & -0.33 & -3.75 & 0.00 & -0.39 & -5.43 & 0.00 \\
\hline Nonjudge & 0.20 & 2.32 & 0.02 & -0.26 & -2.85 & 0.01 & -0.29 & -3.97 & 0.00 \\
\hline Nonreact & 0.14 & 1.83 & 0.07 & -0.14 & -1.66 & 0.10 & -0.25 & -3.68 & 0.00 \\
\hline$R^{2}(\%)$ & \multicolumn{3}{|c|}{44} & \multicolumn{3}{|c|}{38} & \multicolumn{3}{|c|}{57} \\
\hline \multicolumn{10}{|l|}{ Step 2} \\
\hline Observe & 0.03 & 0.35 & 0.72 & 0.04 & 0.45 & 0.66 & 0.05 & 0.85 & 0.40 \\
\hline Describe & -0.13 & -0.15 & 0.88 & 0.10 & 1.03 & 0.31 & 0.02 & 0.31 & 0.76 \\
\hline Actaware & 0.26 & 3.26 & 0.00 & -0.20 & -2.34 & 0.02 & -0.18 & -2.78 & 0.01 \\
\hline Nonjudge & -0.12 & -1.37 & 0.17 & 0.05 & 0.56 & 0.58 & -0.02 & -0.21 & 0.84 \\
\hline Nonreact & 0.01 & 0.15 & 0.88 & 0.01 & 0.14 & 0.89 & -0.11 & -1.90 & 0.06 \\
\hline Alexithymia & 0.01 & 0.12 & 0.91 & 0.06 & 0.49 & 0.62 & -0.01 & -0.09 & 0.93 \\
\hline Acceptance & 0.33 & 3.07 & 0.00 & -0.45 & -3.88 & 0.00 & -0.14 & -1.55 & 0.13 \\
\hline Neuroticism & -0.39 & -3.36 & 0.00 & 0.20 & 1.55 & 0.12 & 0.55 & 5.85 & 0.00 \\
\hline Openness & -0.10 & -1.22 & 0.23 & -0.13 & -1.48 & 0.14 & -0.08 & -1.28 & 0.20 \\
\hline$R^{2}(\%)$ & \multicolumn{3}{|c|}{60} & \multicolumn{3}{|c|}{54} & \multicolumn{3}{|c|}{72} \\
\hline
\end{tabular}


and the usefulness of differentiating between facets in examining the relationship between mindfulness and related constructs. The observe facet, however, should be used with caution in a non-meditating sample, given its deviant relationship with the other facets and theoretically related constructs.

\section{Disclosures None.}

Open Access This article is distributed under the terms of the Creative Commons Attribution Noncommercial License which permits any noncommercial use, distribution, and reproduction in any medium, provided the original author(s) and source are credited.

\section{References}

1. Brown KW, Ryan RM (2003) The benefits of being present: mindfulness and its role in psychological well-being. J Pers Soc Psychol 84:822-848

2. Baer RA, Smith GT, Hopkins J, Krietemeyer J, Toney L (2006) Using self-report assessment methods to explore facts of mindfulness. Assessment 13:27-45

3. Kabat-Zinn J (1990) Full catastrophe living: using the wisdom of your body and mind to face stress, pain and illness. Delacorte, New York

4. Segal ZV, Williams JMG, Teasdale JD (2002) Mindfulness-based cognitive therapy for depression: a new approach to preventing relapse. Guilford, New York

5. Linehan M (1993) Cognitive-behavioral treatment of borderline personality disorder. Guilford, New York

6. Linehan M (1993) Skills training manual for treating borderline personality disorder. Guilford, New York

7. Hayes SC, Strosahl K, Wilson KG (1999) Acceptance and commitment therapy. Guilford, New York

8. Roemer L, Orsillo S, Salters-Pedneault K (2008) Efficacy of an acceptance-based behavior therapy for generalized anxiety disorder: evaluation in a randomized controlled trial. J Consult Clin Psychol 76:1083-1089

9. Ma SH, Teasdale JD (2004) Mindfulness-based cognitive therapy for depression: replication and exploration of differential relapse prevention effects. J Consult Clin Psychol 72:31-40

10. Baer RA (2003) Mindfulness training as a clinical intervention: a conceptual and empirical review. Clin Psychol Sci Pract 10:125-143

11. Grossman P, Niemann L, Schmidt S, Walach H (2004) Mindfulness-based stress reduction and health benefits. A metaanalysis. J Psychosom Res 57:35-43

12. Shigaki CL, Glass B, Schopp LH (2006) Mindfulness-based stress reduction in medical settings. J Clin Psychol Med Settings 13:209-216

13. Teasdale JD, Segal ZV, Williams JMG, Ridgeway VA, Soulsby JM, Lau MA (2000) Prevention of relapse/recurrence in major depression by mindfulness-based cognitive therapy. J Consult Clin Psychol 68:615-623

14. Bohlmeijer ET, Prenger R, Taal E, Cuijpers P (2010) The effects of mindfulness-based stress reduction therapy on mental health of adults with a chronic medical disease: a meta-analysis. J Psychosom Res 68(6):539-544. doi:10.1016/j.jpsychores

15. Buchheld N, Grossman P, Walach H (2001) Measuring mindfulness in insight meditation (vipassana) and meditation-based psychotherapy: the development of the Freiburg Mindfulness Inventory (FMI). J Medit Medit Res 1:11-34

16. Baer RA, Smith GT, Allen KB (2004) Assessment of mindfulness by self-report: the Kentucky Inventory of Mindfulness Skills. Assessment 11:191-206

17. Hayes AM, Feldman G (2004) Clarifying the construct of mindfulness in the context of emotion regulation and the process of change in therapy. Clin Psychol Sci Pract 11:255-262

18. Chadwick P, Hember M, Symes J et al (2008) Responding mindfully to unpleasant thoughts and images: reliability and validity of the Southampton Mindfulness Questionnaire (SMQ). Br J Clin Psychol 47(Pt 4):451-455

19. Baer RA, Smith GT, Lykins E, Button D, Krietemeyer J, Sauer S, Walsh E, Duggan D, Williams JMG (2008) Construct validity of the five facet mindfulness questionnaire in meditating and nonmeditating samples. Assessment 15:329-342

20. Kaplan KH, Goldenberg DL, Galvin-Nadeau M (1993) The impact of a meditation-based stress reduction program on fibromyalgia. Gen Hosp Psychiatry 15:284-289

21. Goldenberg DL, Kaplan KH, Nadeau MG, Brodeur C, Smith S, Schmid CH (1994) A controlled study of a stress-reduction, cognitive-behavioral treatment in fibromyalgia: a randomized controlled trial. J Musculoskelet Pain 2:53-66

22. Grossman P, Tiefenthaler-Gilmer U, Raysz A, Kesper U (2007) Mindfulness training as an intervention for fibromyalgia: evidence of postintervention and 3-year follow-up benefits in well-being. Psychother Psychosom 76:226-233

23. Sephton SE, Salmon P, Weissbecker I, Ulmer C, Floyd A, Hoover K, Studts JL (2007) Mindfulness meditation alleviates depressive symptoms in women with fibromyalgia: results of a randomized clinical trial. Arthritis Rheum 57:77-85

24. Jansen H, Hak T (2005) The productivity of the three-step testinterview (TSTI) compared to an expert review of a self-administered questionnaire on alcohol consumption. J Off Stat 21:103-120

25. Jacobs N, Kleen M, De Groot F, A-Tjak J (2008) Het meten van experiëntiële vermijding. De nederlandstalige versie van de Acceptance and Action Questionnaire-II (AAQ-II). Gedragstherapie 41:349-361

26. Costa PTJ, McCrae RR (1992) Revised Neo Personality Inventory (NEO-PI-R) and NEO Five-Factor Inventory: professional manual. Psychological Assessment Resources, Odessa

27. Hoekstra HA, Ormel H, de Fruyt F (1996) NEO persoonlijkheidsvragenlijsten: NEO-PI-R \& NEO-FFI [NEO personality questionnaires: NEO-PI-R \& NEO-FFI]. Swets \& Zeitlinger, Lisse

28. Murray G, Rawlings D, Allen NB, Trinder J (2003) NEO fivefactor inventory scores: psychometric properties in a community sample. Meas Eval Couns Dev 36:140-149

29. Bagby RM, Parker JDA, Taylor GJ (1994) The twenty-item Toronto Alexithymia Scale: part I, item selection and crossvalidation of the factor structure. J Psychosom Res 38:23-32

30. Bagby RM, Taylor GJ, Parker JD (1994) The twenty-item Toronto Alexithymia Scale-II. Convergent, discriminant, and concurrent validity. J Psychosom Res 38:33-40

31. Kooiman CG, Spinhoven P, Trijsburg RW (2002) The assessment of alexithymia: a critical review of the literature and a psychometric study of the Toronto Alexithymia Scale-20. J Psychosom Res 53:1083-1090

32. Zigmond AS, Snaith RP (1983) The Hospital Anxiety and Depression Scale. Acta Psychiatr Scand 67:361-370

33. Spinhoven PH, Ormel J, Sloekers PPA, Kempen GIJM, Speckens AEM, Van Hemert AM (1997) A validation study of the Hospital Anxiety and Depression Scale (HADS) in different groups of Dutch subjects. Psychol Med 27:363-370

34. Ware JEJ, Kosinski M, Keller SD (1996) A 12 item short form health survey: construction of scales and preliminary tests of reliability and validity. Med Care 34:220-233 
35. Ware JE, Kosinski M, Keller SD (1995) How to score the SF-12 physical and mental health summary scales. The Health Institute, New England Medical Center, Boston

36. Gandek B, Ware JE, Aaronson NK, Apolone G, Bjorner JB, Brazier JE et al (1998) Cross-validation of item selection and scoring for the SF-12 Health Survey in nine countries: results from the IQOLA project. International Quality of Life Assessment. J Clin Epidemiol 51:1171-1178

37. Hu LT, Bentler PM (1998) Fit indices in covariance structure modeling: sensitivity to underparameterized model misspecification. Psychol Methods 3:424-453

38. Browne MW, Cudeck R (1993) Alternative ways of assessing model fit. In: Bollen KA, Long JS (eds) Testing structural equation models. Sage Publications, Newbury Park, pp 136162

39. Hu LT, Bentler PM (1999) Cutoff criteria for fit indexes in covariance structure analysis: conventional criteria versus new alternatives. Struct Equ Modeling 6:1-55

40. Cicchetti DV (1994) Guidelines, criteria, and rules of thumb for evaluating normed and standardized assessment instruments in psychology. Psychol Assess 6:284-290

41. Cohen J (1988) Statistical power analysis for the behavioral sciences, 2nd edn. Lawrence Erlbaum, New Jersey

42. Ford J, MacCallum R, Tait M (1986) The application of factor analysis in psychology: a critical review and analysis. Pers Psychol 39:291-314 